\title{
Tunnelling in low-temperature hydrogen-atom and proton transfers
}

\author{
Luis G. Arnaut*, Sebastião J. Formosinho, Monica Barroso \\ Departamento de Química, Universidade de Coimbra, P-3049 Coimbra Codex, Portugal \\ Received 8 August 2005; received in revised form 23 September 2005; accepted 3 October 2005 \\ Available online 21 November 2005
}

\begin{abstract}
The reaction path of the interacting-state model with the Lippincott-Schroeder potential for hydrogen bonds, is used in transition-state theory calculations with the semiclassical correction for tunnelling (LS-ISM/scTST) to estimate proton and hydrogen-atom transfer rates at low temperatures. Down to $100 \mathrm{~K}$, the semiclassical correction leads to semi-empirical rates and isotope effects that are in good agreement with the thermal tautomerism of porphine, and the excited-state tautomerisms of salicylideneanilines and 2-( $2^{\prime}$-hydroxyphenyl)benzoxazole. For lower temperatures, the tunnelling corrections become extremely high and unreliable. It is shown that the permeability of an Eckart barrier fitted to the curvature of the LS-ISM reaction path leads to good estimates of these reaction rates down to $2 \mathrm{~K}$.
\end{abstract}

(C) 2005 Elsevier B.V. All rights reserved.

Keywords: Tunnelling; Proton transfer; Hydrogen atom transfer; Tautomerism

\section{Introduction}

Hydrogen-atom and proton transfer reactions are among the most fundamental processes in Chemistry and are involved in many biochemical transformations. Although their mechanisms have been thoroughly investigated, the quantification of the role of nuclear tunnelling in these reactions remains a challenge. Bell pioneered the study of nuclear tunnelling, and classified the magnitude of tunnelling corrections $(\kappa)$ in three groups [1]: negligible tunnelling $(1<\kappa<1.1)$, small to moderate tunnelling $(1.1<\kappa<4)$, and large tunnelling $(\kappa>$ 4). This last group includes intramolecular hydrogen-atom and proton transfer reactions in low-temperature matrices. Clearly, any method to calculate the rates of such reactions at low temperatures will require accurate estimates of the tunnelling corrections. In this work, we use the semiclassical approximation to tunnelling in the formalism of the transition-state theory (scTST) [2], with the reaction path of the Intersecting/ Interacting state model $[3,4]$ including the hydrogen-bonding potential of Lippincott and Schroeder [5], to calculate the rates of intramolecular reactions at cryogenic temperatures. Alternatively, fitting an Eckart barrier to the same reaction path and calculating its permeability to a particle of a given energy, can also be used to estimate the reaction rate, LS-ISM/Eckart. We

\footnotetext{
* Corresponding author. Tel.: +351 239854484; fax: +351 239827703.

E-mail address: lgarnaut@ci.uc.pt (L.G. Arnaut).
}

0022-2860/\$ - see front matter (C) 2005 Elsevier B.V. All rights reserved. doi:10.1016/j.molstruc.2005.10.002 assess the performance of both procedures using the LS-ISM reaction paths of thermal and photochemical $\mathrm{H}$-atom/proton transfers for which experimental rates are also available for comparison.

The systems selected for this study are the thermal tautomerism of porphine, the singlet-state proton transfer occurring in salicylideneanilines and the triplet-state proton/ hydrogen-atom transfer in 2-(2'-hydroxyphenyl)benzoxazole. Porphyrins and their derivatives are very important for their involvement in biological systems and their application in molecular electronics and non-linear optical materials. The thermal tautomerism rates of the simplest member of this family, the free-base porphyrin $\left(\mathrm{H}_{2} \mathrm{P}\right)$, and its deuterated analogue $\left(D_{2} P\right)$, have been studied in the $95-309 \mathrm{~K}$ temperature range $[6,7]$, and recently attracted considerable theoretical interest [8-10]. These data offer the opportunity to test the role of tunnelling in a thermal $\mathrm{H}$-atom transfer reaction studied over a large range of temperatures. Salicylideneanilines are aromatic Schiff bases that undergo ultrafast excited-state intramolecular proton transfer (ESIPT) from the first singlet state. They are very popular because of their thermo and photochromic properties [11]. ESIPT in 4-methyl- $N$-salicylideneaniline (MSA), 4-methyl- $N-5^{\prime}$-chlorosalicylideneaniline (MClSA) and $\mathrm{N}$-5'-chlorosalicylideneaniline (ClSA), are weakly exothermic and, at $77 \mathrm{~K}$, give rise to rates that are lower than the vibrational mode of the intramolecular H-bond [12]. Thus, these ESIPT involve a barrier and are particularly well suited to evaluate tunnelling in ultrafast ESIPT. Finally, Grellmann and co-workers made a detailed study of the 
keto-enol tautomerism established within the lifetime of the triplet state of 2-(2'-hydroxyphenyl)benzoxazole (HBO), and measured their proton/hydrogen-atom transfer rates between 2 and $220 \mathrm{~K} \mathrm{[13-16].} \mathrm{This} \mathrm{triplet-state} \mathrm{reaction} \mathrm{is} \mathrm{most}$ interesting because it is nearly isothermic [17], but it is several orders of magnitude slower than the analogous singlet-state reaction.

The molecular structures of reactants and products in $\mathrm{H}$-atom and proton transfer intramolecular reactions are often written in identical forms. It is however important to distinguish both types of reactions. In an intramolecular proton transfer, either the reactant or the product has a zwitterionic character. In an intramolecular $\mathrm{H}$-atom transfer, the reactant and the product are of biradicaloid character. Scheme 1 illustrates this difference for the tautomerisms of $\mathrm{HBO}$ and MSA, under the assumption that the triplet state reaction is an $\mathrm{H}$-atom transfer, but the singlet state one is a proton transfer [18]. Because a zwitterionic tautomer is formed, or destroyed, in a proton transfer process, it does not require rearrangement of the electronic charge distribution, which is the case of $\mathrm{H}$-atom transfer [19]. The heterolytic or homolytic cleavage of the A-H bond is probably related both with the multiplicity as well as the $\left(n, \pi^{*}\right)$ or $\left(\pi, \pi^{*}\right)$ nature of the electronic states involved.

Accurate methods to evaluate tunnelling corrections for a given potential energy surface have been available for some time [20]. However, for the large molecular systems addressed in this work, it is impractical to build potential energy surfaces from first principles and evaluate multidimensional tunnelling corrections. Instead, we use the semiclassical approximation to the tunnelling correction [2], and apply it to the reaction path of LS-ISM. We have shown that this reaction path, when combined with scTST, can be used to make absolute rate calculations for atom transfers in the gas phase and for proton transfers in solution and in enzymes that are in very good agreement with the experimental data [3,21]. However, at low temperatures, small errors in the reaction path have dramatic effects in the reaction rates. For example, at room temperature, a $1 \mathrm{kcal} / \mathrm{mol}$ error in the barrier height corresponds to a factor of 5 in the rate, whereas at $77 \mathrm{~K}$ it leads to a factor of 700 .

In the spirit of the earlier work with ISM $[3,4]$, our reaction path calculations only employ information on the reactants and products, including the reaction energy, and do not involve the fitting of any parameters to the kinetic data. In this sense, ISM can be classified as a semi-empirical method.

\section{The LS-ISM method}

The ISM method has been described in detail elsewhere [3, 4]. Briefly, the classical reaction path of ISM for a reaction of the type (charges omitted)

$\mathrm{AH}+\mathrm{B} \rightarrow \mathrm{A}+\mathrm{HB}$

is a linear interpolation between the Morse curves of HA and $\mathrm{HB}$ along the reaction coordinate

$V_{\mathrm{cl}}(n)=(1-n) V_{\mathrm{HA}}+n V_{\mathrm{HB}}+n \Delta V^{0}$

where $\Delta V^{0}$ is the classical reaction energy and the reaction coordinate $n\left(=n_{\mathrm{HB}}\right)$ is the bond order of the new bond formed in the course of the reaction [22]. The Morse curves are expressed in terms of the bond extensions from equilibrium to transition state configurations

$V_{\mathrm{HA}}=D_{\mathrm{HA}}\left\{1-\exp \left[\frac{-\beta_{\mathrm{HA}}\left(l_{\mathrm{HA}}^{\ddagger}-l_{\mathrm{HA}}\right)}{m}\right]\right\}^{2}$
$V_{\mathrm{HB}}=D_{\mathrm{HB}}\left\{1-\exp \left[\frac{-\beta_{\mathrm{HB}}\left(l_{\mathrm{HB}}^{\ddagger}-l_{\mathrm{HB}}\right)}{m}\right]\right\}^{2}$

and these are related to the corresponding transition state bond orders

$l_{\mathrm{HA}}^{\ddagger}-l_{\mathrm{HA}}=-a^{\prime}\left(l_{\mathrm{HA}}+l_{\mathrm{HB}}\right) \ln \left(n_{\mathrm{HA}}^{\ddagger}\right)$
$l_{\mathrm{HB}}^{\ddagger}-l_{\mathrm{HB}}=-a^{\prime}\left(l_{\mathrm{HA}}+l_{\mathrm{HB}}\right) \ln \left(n_{\mathrm{HB}}^{\ddagger}\right)$

where $a^{\prime}=0.182$, which is reminiscent of Pauling's relation
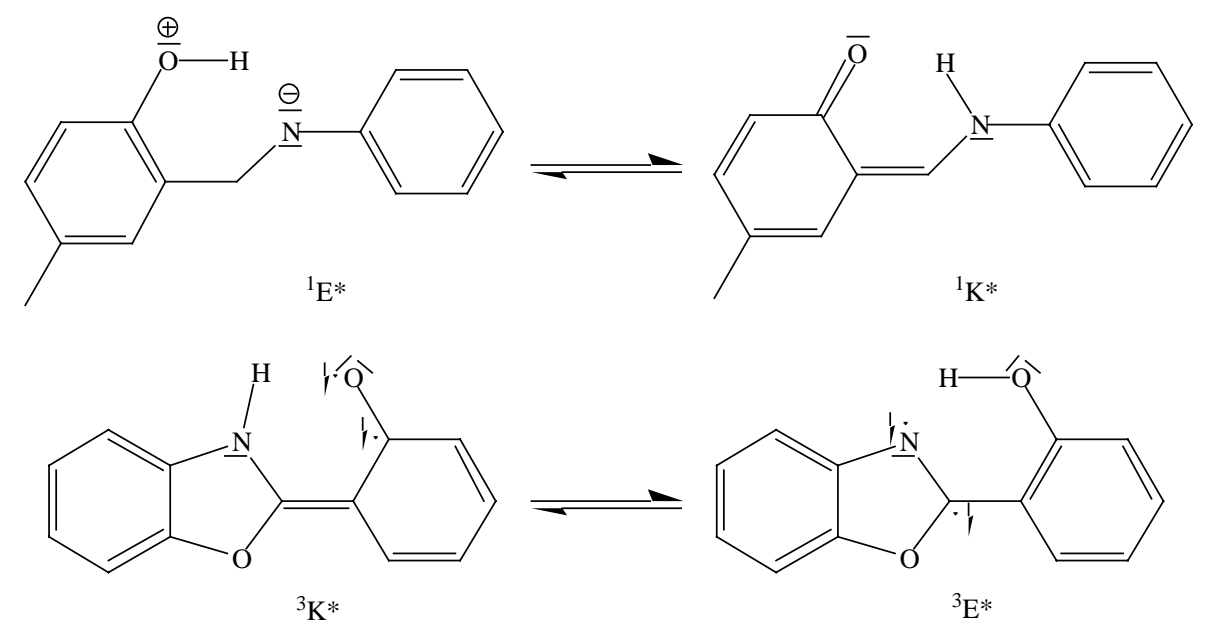

${ }^{3} \mathrm{E}^{*}$

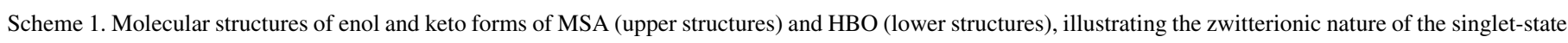
reaction and the biradicaloid nature of the triplet state reaction. 
between bond lengths and bond orders [23]. The parameter $m$ is equal to unity in the reactants and products, but it is multiplied by a switching function to attain the value of the electrophilicity index of Parr [24] at the transition state. The electrophilicity index is the ratio between the negative of the electronic chemical potential, $\mu_{\mathrm{el}}$, and the chemical hardness, $\eta$,

$m=\frac{-\mu_{\mathrm{el}}}{\eta}=\frac{I_{\mathrm{P}}+E_{\mathrm{A}}}{I_{\mathrm{P}}-E_{\mathrm{A}}}$

where $I_{\mathrm{P}}$ is the ionisation potential and $E_{\mathrm{A}}$ is the electron affinity of A or B. It gives the saturation point for electron inflow in the transition state. For the excited-state reactions, the ionisation potential is calculated from $I_{\mathrm{P}}^{*}=I_{\mathrm{P}}-E^{*}$, where $E^{*}$ is the excited-state energy [25].

The vibrationally-adiabatic path is calculated adding the difference in ZPE to the classical energy at each point along the reaction path,

$V_{\mathrm{ad}}(n)=V_{\mathrm{cl}}(n)+\sum_{i}\left(\frac{1}{2} h c \bar{\nu}_{i}\right)$

where $\bar{\nu}_{i}$ are the vibration frequencies of the normal modes orthogonal to the reaction coordinate. We estimate the frequencies of the triatomic transition state from Wilson's equation with the neglect of the interaction between bending and stretching [26], using fractional bonds in the $\{\mathrm{A} \cdots \mathrm{H} \cdots \mathrm{B}\}^{*}$ transition state and the same switching function as that employed for $m$, to provide the correct asymptotic limits. The linear relation between symmetric stretching and bending frequencies in triatomic systems is employed to estimate the bending frequency from the symmetric stretching frequency. In earlier applications of the ISM/scTST method, the transition state was assumed to be a linear triatomic molecule. However, the transition state of intramolecular reactions is bent, and has one bending frequency less. We account for this difference in the application of the method to intramolecular reactions and include only one bending frequency at the transition state. The consequence of this change is just a decrease in the vibrationally-adiabatic barrier $\left(\Delta V_{\mathrm{ad}}^{\ddagger}\right)$ by a few tenths of a $\mathrm{kcal} / \mathrm{mol}$.

The presence of H-bonds in the reaction co-ordinate is taken into account using the Lippincott-Schroeder (LS) potential [5]. The LS potential relates the $\mathrm{AB}$ distance in the molecule $\left(l_{\mathrm{AB}}\right)$, with its $\mathrm{H}$-bond energy $\left(D_{\mathrm{OAB}}\right)$ and the vibrational frequency of the H-bond stretching $\left(\varpi_{\mathrm{AB}}\right)$. For example, the near-neighbour $\mathrm{H}_{2} \mathrm{O} \cdots \mathrm{H}_{2} \mathrm{O}$ distance in the tetrahedral network of pure water is $l_{\text {OOwater }}=2.85 \AA$ [27], the enthalpy of this H-bond is $2.53 \pm$ $0.10 \mathrm{kcal} / \mathrm{mol}$ [28], and the vibration frequency of an oxygen atom against its nearest-neighbor oxygen is $\varpi_{\text {OOwater }}=$ $170 \mathrm{~cm}^{-1}$ [29]. The LS potential gives $l_{\text {OOwater }}=2.80 \AA$, $D_{0 \text { (water) }}=2.0 \mathrm{kcal} / \mathrm{mol}$ and $\varpi_{\text {OOwater }}=163 \mathrm{~cm}^{-1}$. This last value can also be regarded as the vibrational frequency of the $\mathrm{H}$-bond stretching. The advantage of including the LS potential in the ISM reaction coordinate is that it requires experimental information on only one of the parameters to estimate the other two and obtain the Morse potential representing the AB bond.
For example, only the $l_{\mathrm{OO}}\left(\mathrm{H}_{3} \mathrm{O}^{+} \cdots \mathrm{OH}_{2}\right)$ distance in water is known, $l_{\mathrm{O}^{+} \mathrm{O}}=2.56 \AA$ [27], and the LS potential with $l_{\mathrm{O}^{+} \mathrm{O}}=$ $2.56 \AA$ gives $D_{0\left(\mathrm{O}^{+} \mathrm{HO}\right)}=7.0 \mathrm{kcal} / \mathrm{mol}$ and $\varpi_{\mathrm{O}^{+} \mathrm{HO}}=500 \mathrm{~cm}^{-1}$. The LS potential clearly shows that the presence of charges increases the strength of the H-bond, and this is taken into account for species of zwitterionic character. For the intramolecular reactions addressed in this work, the $\mathrm{AB}$ distances can be conveniently obtained from the crystal structures of the molecules. Lippincott and Schroeder also offered a procedure to include bent $\mathrm{H}$-bonds in their potential [30]. However, such a refinement is not significant for the molecular systems addressed in this work, and it is not included in the potential functions.

The AB bond distances, dissociation energies and vibrational frequencies given by the LS potential are used to construct Morse curves for the AB bond. The minimum of this Morse curve gives the location of the reactant state as a hydrogen-bonded species. This species is regarded as an incipient proton transfer, with an $\mathrm{H} \cdots \mathrm{B}$ bond order larger than zero $\left(n_{\mathrm{HB}}^{*}>0\right)$, i.e. the reaction coordinate is advanced with respect to the isolated reactants. Similarly, for the products, the bond order does not completely nullify, $n_{\mathrm{HA}}^{*}<1$, and the conservation of bond order requires that $n^{*}=$ $1-n_{\mathrm{HA}}^{*}>0$. The proton-transfer reaction coordinate in the region between the precursor and successor $\mathrm{H}$-bonded complexes is a combination of the ISM potential with the repulsion of the Morse $\mathrm{AB}$ potential

$$
\begin{aligned}
& V_{\mathrm{cl}}\left(n_{\mathrm{HB}}^{*}<n<n^{*}\right) \\
& =(1-j) V_{\mathrm{HA}}(j)+j V_{\mathrm{HB}}(j)+k \Delta V^{0}-D_{\mathrm{e}, \mathrm{AB}}+k\left(D_{\mathrm{e}, \mathrm{AB}}-D_{\mathrm{e}, \mathrm{BA}}\right)
\end{aligned}
$$

where $D_{\mathrm{e}, \mathrm{AB}}$ and $D_{\mathrm{e}, \mathrm{BA}}$ are the electronic dissociation energies of the $\mathrm{A}-\mathrm{H} \cdots \mathrm{B}$ and $\mathrm{A} \cdots \mathrm{H}-\mathrm{B}$ hydrogen bonds, respectively and the transformation of coordinates

$$
k=\frac{\left(n-n_{\mathrm{HB}}^{*}\right)}{\left(1-n_{\mathrm{HA}}^{*}-n_{\mathrm{HB}}^{*}\right)}
$$

$j=n-n_{\mathrm{HB}}^{*}$ for $n \leq n^{\ddagger}$

$j=n+n_{\mathrm{HB}}^{*}$ for $n>n^{*}$

translates the contraction of the reaction coordinate. This transformation and the search for a transition state location where $\left|V_{\mathrm{cl}}\left(n^{\ddagger}-\Delta n\right)-V_{\mathrm{cl}}\left(n^{\ddagger}+\Delta n\right)\right|<10 \mathrm{cal} / \mathrm{mol}$ with a bondorder step of $\Delta n=1 / 600$, guarantees the continuity of the reaction coordinate.

The reaction coordinate of $\mathrm{H}$-atom transfers has, in addition to the contributions expressed by Eq. (7), a contribution from the repulsive component of the $\mathrm{AB}$ bond Morse curve, $V_{\mathrm{AB}}$ or $V_{\mathrm{BA}}$, 


$$
\begin{aligned}
& V_{\mathrm{cl}}\left.n_{\mathrm{HB}}^{*}<n<n^{\ddagger}\right) \\
&= V_{\mathrm{AB}}(n)+(1-j) V_{\mathrm{HA}}(j)+j V_{\mathrm{HB}}(j)+k \Delta V^{0} \\
&-D_{\mathrm{e}, \mathrm{AB}}+k\left(D_{\mathrm{e}, \mathrm{AB}}-D_{\mathrm{e}, \mathrm{BA}}\right) \\
& V_{\mathrm{cl}}\left(n^{\ddagger}<n<n_{\mathrm{HA}}^{*}\right) \\
&=V_{\mathrm{BA}}(n)+(1-j) V_{\mathrm{HA}}(j)+j V_{\mathrm{HB}}(j)+k \Delta V^{0} \\
& \quad-D_{\mathrm{e}, \mathrm{AB}}+k\left(D_{\mathrm{e}, \mathrm{AB}}-D_{\mathrm{e}, \mathrm{BA}}\right)
\end{aligned}
$$

This contribution is absent in proton transfers because the transfer of the positive charge quenches the repulsion associated with the overlap of the electronic wavefunctions of A and B [4].

The movement of the $\mathrm{H}$-atom, or proton, along the intramolecular $\mathrm{H}$-bond determines the reaction frequency of $\mathrm{H}$-bonded intramolecular reactions. We obtain this frequency from the H-bond stretching frequency, $\varpi_{\mathrm{AB}}$, which, for the case of water, has also been described as a restricted translation of the $\mathrm{H}_{2} \mathrm{O}$ molecules along the $\mathrm{O}-\mathrm{H} \cdots \mathrm{O}$ direction [29]. Then, the pre-exponential factor of our rate calculations becomes the product between the semiclassical tunnelling correction, $\kappa(T)$, and $c \varpi_{\mathrm{AB}}$,

$k_{\mathrm{ISM}}=\kappa(T) c \varpi_{\mathrm{AB}} \exp \left(-\frac{\Delta V_{\mathrm{ad}}^{\ddagger}}{R T}\right)$

In summary, the ISM/scTST method applied in this work is identical to its more recent formulation [4,21], except for the pre-exponential factor of Eq. (10), a minor change in the zeropoint energy of the transition state, and the use of the experimental reaction energies, $\Delta V_{\mathrm{ad}}^{0}$.

The differences between ISM/scTST calculations on $\mathrm{H}$-atom $v s$ proton transfers, already addressed in earlier work $[4,25]$, need to be re-emphasised. First, the ISM reaction path for $\mathrm{H}$-atom transfers between hydrogen-bonded species contains the repulsive part of the Morse potential estimated by the LS potential, but for proton transfers that repulsion is not included because a positive charge is always maintained between the electronic wavefunctions of A and B. Second, the ionisation potential of $\mathrm{H}$-atom transfers it is simply the $I_{\mathrm{P}}$ of the radical or of the molecule, but for ESIPT it is estimated from $I_{\mathrm{P}}^{*}=I_{\mathrm{P}}-E^{*}$, where $I_{\mathrm{P}}$ is the ionisation potential of the radical A or, in its absence, of the molecule HA. This latter difference is motivated by the use of the electronic excitation to form biradicals in $\mathrm{H}$-atom transfers, and to achieve a charge separation in ESIPT.

\section{Tunnelling corrections}

According to the semiclassical approximation to tunnelling corrections, the transmission coefficient and the transmission probabilities are [2],

$\kappa(T)=1+\frac{2}{k_{B} T} \int_{\varepsilon_{0}}^{\Delta V_{a d}^{\ddagger}} \sinh \left(-\frac{\Delta V_{a d}^{\ddagger}-\varepsilon}{k_{B} T}\right) G(\varepsilon) \mathrm{d} \varepsilon$

$$
G(\varepsilon)=\left\{\begin{array}{cc}
\{1+\exp [2 \gamma(\varepsilon)]\}^{-1} & \varepsilon_{0} \leq \varepsilon \leq \Delta V_{a d}^{\ddagger} \\
1-G\left(2 \Delta V_{a d}^{\ddagger}-\varepsilon\right) & \Delta V_{a d}^{\ddagger} \leq \varepsilon \leq 2 \Delta V_{a d}^{\ddagger}-\varepsilon_{0} \\
1 & 2 \Delta V_{a d}^{\ddagger}-\varepsilon_{0}<\varepsilon
\end{array}\right.
$$

respectively, where the barrier penetration integral is given by

$\gamma(\varepsilon)=\frac{2 \pi}{h} \int_{s_{<}}^{s_{>}} \sqrt{2 \mu\left[V_{\mathrm{ad}}(s)-\varepsilon\right]} \mathrm{d} s \quad \varepsilon<\Delta V_{\mathrm{ad}}^{\ddagger}$

and the reduced mass $\mu$ is that of the antisymmetric stretch, calculated assuming identical force constants for $\mathrm{AH}$ and $\mathrm{HB}$ at the transition state,

$\mu=\frac{2}{\mu_{\mathrm{A}}+2 \mu_{\mathrm{H}}+\mu_{\mathrm{B}}+\sqrt{\left(\mu_{\mathrm{A}}-\mu_{\mathrm{B}}\right)^{2}+4 \mu_{\mathrm{H}}^{2}}}$

where $\mu_{\mathrm{i}}=m_{\mathrm{i}}^{-1}$. The threshold energy in Eqs. (11-12) is the limiting value of the vibrationally-adiabatic potential energy for exothermic or endothermic reactions,

$\varepsilon_{0}=\max \left[V_{\mathrm{ad}}(s=-\infty), V_{\mathrm{ad}}(s=+\infty)\right]$

where $s_{>}$and $s_{<}$are the classical turning points, i.e., the locations at which $V_{\mathrm{ad}}(s)=\varepsilon$.

The vibrationally-adiabatic path is expressed in terms of the $\mathrm{H}-\mathrm{B}$ bond order $n$ and in terms of the reaction co-ordinate $s$. The two are related by Eq. (4) and

$s=-\sqrt{\left(l_{A H}-l_{A H}^{\ddagger}\right)^{2}+\left(l_{B H}-l_{B H}^{\ddagger}\right)^{2}} \quad l_{A H} \leq l_{A H}^{\ddagger}$

$s=+\sqrt{\left(l_{A H}-l_{A H}^{\ddagger}\right)^{2}+\left(l_{B H}-l_{B H}^{\ddagger}\right)^{2}} \quad l_{B H}>l_{B H}^{\ddagger}$

$\mathrm{H}$-atom or proton transfers between heavy atoms often lead to vibrationally-adiabatic paths with two maxima. Formally, the resolution of this problem should be made in the framework of the canonically unified theory [31]. In practice, given the approximate nature of our treatment, it suffices to use TST with $\Delta V_{\text {ad }}^{\ddagger}$ equal to the value of the highest maximum. In these cases, the tunnelling correction of the particles with energies between that of the highest maximum and the minimum between the two maxima is calculated for the highest barrier only.

It is obvious that the exponential nature of Eq. (10) will make insignificant any rate at sufficiently low temperatures, even for a barrier of a few $\mathrm{kcal} / \mathrm{mol}$. The tunnelling correction of Eq. (11) varies in a opposite manner, but the uncertainties associated with the product of very low and very high number will ultimately render the semiclassical approximation useless at very low temperatures. Alternatively, rather than integrating over a range of Boltzmann-averaged energies, we can consider that the initial system has a definite energy $W=k_{\mathrm{B}} T$ above the reactants vibrationally-adiabatic energy. Eckart [32] has given a solution for the Schroedinger equation when a particle of mass $\mu$ and total energy $W$ collides with a one-dimensional barrier of the form

$V(x)=-\frac{A y}{1-y}-\frac{B y}{(1-y)^{2}}$ 
$y=-\exp \left(\frac{2 \pi x}{l}\right)$

where

$A=\Delta V_{\mathrm{ad}}^{\ddagger}-\left(\Delta V_{\mathrm{ad}}^{\ddagger}-\Delta V_{\mathrm{ad}}^{0}\right)$

$B=\left[\sqrt{\Delta V_{\mathrm{ad}}^{\ddagger}}+\sqrt{\Delta V_{\mathrm{ad}}^{\ddagger}-\Delta V_{\mathrm{ad}}^{0}}\right]^{2}$

$l=2 \pi \sqrt{-\frac{2}{f_{\mathrm{ad}}}}\left[\frac{1}{\sqrt{\Delta V_{\mathrm{ad}}^{\ddagger}-\Delta V_{\mathrm{ad}}^{0}}}+\frac{1}{\sqrt{\Delta V_{\mathrm{ad}}^{\ddagger}}}\right]^{-1}$

Eckart's solution is

$\kappa(W)=1-\frac{\cosh [2 \pi(\alpha-\beta)]+\cosh (2 \pi \delta)}{\cosh [2 \pi(\alpha+\beta)]+\cosh (2 \pi \delta)}$

where

$\alpha=\frac{1}{2} \sqrt{\frac{W}{C}}$

$\beta=\frac{1}{2} \sqrt{\frac{W-A}{C}}$

$\delta=\frac{1}{2} \sqrt{\frac{B-C}{C}}$

$C=\frac{h^{2}}{8 \mu l}$

and can be readily calculated when the asymptotic limits of the potential are known, as well as its curvature at the maximum, $f_{\text {ad }}$. The Eckart barrier can be used to simulate the vibrationally-adiabatic LS-ISM reaction path, when $f_{\mathrm{ad}}$ is taken from the inverted parabola that fits the curvature of this path. The minima just before and after $\Delta V_{\text {ad }}^{\ddagger}$ are used to fit the inverted parabola and obtain the curvature, as illustrated in

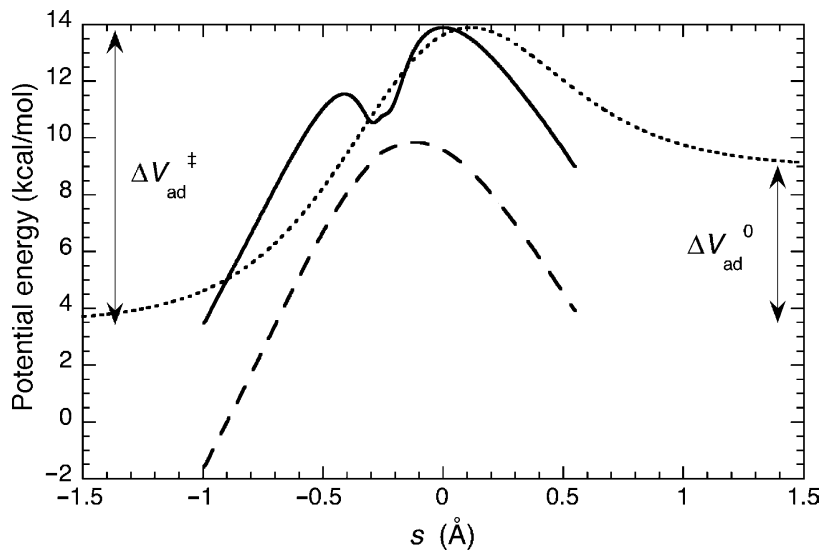

Fig. 1. Vibrationally-adiabatic (full line) and classical (dashed line) LS-ISM reaction paths for proton and $\mathrm{H}$-atom transfers using the data on $\mathrm{H}_{2} \mathrm{P}$. The dotted line represents the Eckart barrier with the asymptotic limits and curvature at the transition sate of the vibrationally-adiabatic path.
Fig. 1 for the reaction path of $\mathrm{H}_{2} \mathrm{P}$, obtained with the data on the reactants and products discussed further below.

The tunnelling rate can now be expressed as the product between the frequency of impact in the barrier and its permeability $[1,33,34]$

$k_{\mathrm{tun}}=c \varpi \kappa(W)$

This expression can be employed directly when $\Delta V_{\mathrm{ad}}^{0} \leq 0$, on the assumption that the system can be described by particle of mass $\mu$ and energy $W=k_{\mathrm{B}} T$. For endothermic reactions, the particle can only tunnel if it has at least an energy of $\Delta V_{\mathrm{ad}}^{0}$. Thus, the tunnelling rate of endothermic reactions must be multiplied by $\exp \left(-\Delta V_{\mathrm{ad}}^{0} / k_{\mathrm{B}} T\right)$.

The temperature-dependence of the Eckart tunnelling correction is much milder than that of the semiclassical correction. Additionally, it can be obtained analytically and should be more reliable at very low temperatures. We represent the rates calculated by this method as LS-ISM/Eckart.

\section{Results and discussion}

It is now established that the mechanism of $\mathrm{H}_{2} \mathrm{P}$ tautomerization involves the stepwise migration of two hydrogen atoms, with the formation of a metastable cis intermediate. The energy of this intermediate is not known for certainty, but the Arrhenius slope observed at low temperatures places an upper limit of $6.3 \pm 0.9 \mathrm{kcal} / \mathrm{mol}$ to the enthalpy of $c$ is intermediate with respect to that of the trans reactant [8]. MP2 and DFT calculations give classical reaction energies in the $7.6-10.0 \mathrm{kcal} / \mathrm{mol}$ range [9], which seem to be excessive in view of the Arrhenius slope. Smedarchina and coworkers suggest a reaction energy of 5.5-5.7 kcal $/ \mathrm{mol}$ based on their own empirical fits of the kinetic data [8]. We adopt this value and make the vibrationally adiabatic reaction energy $\Delta V_{\text {ad }}^{0}=5.5 \mathrm{kcal} / \mathrm{mol}$ for the $\mathrm{H}_{2} \mathrm{P}$ tautomerization. From the geometry of $\mathrm{H}_{2} \mathrm{P}$ [35], we estimate a distance of $2.81 \AA$ between the neighbouring nitrogen atoms. According to the LS potential, a $2.80 \AA$ distance between nitrogen atoms corresponds to $D_{0(\mathrm{NN})}=1.50 \mathrm{kcal} / \mathrm{mol}\left(D_{\mathrm{e}(\mathrm{NN})}=1.60 \mathrm{kcal} / \mathrm{mol}\right)$. These parameters and the data on pyrrole in Table 1 suffice to perform ISM/scTST calculations. There is no fitting or scaling of any parameters in these calculations. Fig. 2 compares our calculations with the experimental rates [6,7].

With $\Delta V_{\text {ad }}^{0}=5.5 \mathrm{kcal} / \mathrm{mol}$, the LS-ISM $/ \mathrm{scTST}$ rates are in good agreement with the experimental data in the $95-309 \mathrm{~K}$ temperature range. For example, the experimental rate at $300 \mathrm{~K}$ is $k_{\exp }=2 \times 10^{4} \mathrm{~s}^{-1}$ [7], and we calculate $k_{\mathrm{ISM}}=1.8 \times 10^{5} \mathrm{~s}^{-1}$, whereas at $110 \mathrm{~K}$ we compare $1.4 \times 10^{-4} \mathrm{~s}^{-1}$ [6] with $3.3 \times$ $10^{-5} \mathrm{~s}^{-1}$. This last value includes a tunnelling correction of $1.2 \times 10^{4}$. The large tunnelling correction is at the origin of a kinetic isotope effect (KIE) of 56 at $110 \mathrm{~K}$ for $\mathrm{D}_{2} \mathrm{P}$ tautomerization, which is nevertheless smaller than the KIE of 256 experimentally measured [6].

Fig. 2 shows that the reaction rates of the semiclassical approximation remain higher than the tunnelling frequency estimated from the permeability of the Eckart barrier for 
Table 1

Bond lengths, bond dissociation energies, vibrational frequencies of the molecules and ionization potentials and electron affinities of the radicals employed in the calculation of the energy barriers

\begin{tabular}{llllll}
\hline & $l_{\text {eq }}(\AA)$ & $\begin{array}{l}D_{298}^{0} \\
\left(\mathrm{kcal} \mathrm{mol}^{-1}\right)\end{array}$ & $\begin{array}{l}\varpi_{\mathrm{e}} \\
\left(\mathrm{cm}^{-1}\right)\end{array}$ & $I_{\mathrm{P}}(\mathrm{eV})$ & $E_{\mathrm{A}}(\mathrm{eV})$ \\
\hline $\mathrm{C}_{4} \mathrm{H}_{4} \mathbf{N H}$ & 0.996 & $93.9^{\mathrm{a}}$ & 3500 & $8.207^{\mathrm{b}}$ & $2.145^{\mathrm{c}}$ \\
$\mathrm{C}_{6} \mathrm{H}_{5} \mathrm{NH}_{2}$ & 0.998 & 88.0 & 3400 & $7.720^{\mathrm{b}}$ & \\
$\mathrm{C}_{6} \mathrm{H}_{5} \mathbf{O H}$ & 0.956 & 86.5 & 3650 & 8.56 & 2.253 \\
\hline
\end{tabular}

Boldface letters indicate where the radical is centred after the bond to the hydrogen atom is broken; bond lengths and bond dissociation energies from Ref. [36]; ionisation potentials and electron affinities from webbook.nist.gov, except where noted.

a Ref. [37].

b For the molecule.

c Ref. [38].

temperatures above $100 \mathrm{~K}$. Indeed, from the curvature of the vibrationally-adiabatic barrier, $\nu^{\ddagger}=974 \mathrm{i} \mathrm{cm}^{-1}$, we calculate $k_{\text {tun }}=2.8 \times 10^{-7} \mathrm{~s}^{-1}$ at $100 \mathrm{~K}$, one order of magnitude below $k_{\text {exp }}$ and $k_{\text {ISM. }}$. Only for lower temperatures is the permeability of the Eckart barrier relevant for the $\mathrm{H}_{2} \mathrm{P}$ tautomerization rate.

ESIPT reactions are among the fastest chemical transformations and often occur without a barrier [39]. In such cases the rates approach the frequency of the vibrational mode involving the H-bond. For example, the rate of ESIPT in 3-hydroxyflavone is $4.2 \times 10^{12} \mathrm{~s}^{-1}$ [39]. In the salicylideneanilines addressed in this work, the rates are also very fast, but fall below that frequency and should have a barrier. This is probably related to the weak exothermicity of these reactions: $\Delta E^{0}=-0.53,-0.69$ and $-1.01 \mathrm{kcal} / \mathrm{mol}$ for MSA, MClSA and ClSA, respectively [12]. The LS-ISM reaction path for these reactions requires the $\mathrm{OH}$ and $\mathrm{NH}$ bond parameters taken from phenol and aniline, the ionisation potential and electron affinity of the phenoxy radical, all given in Table 1, together with the singlet-state energy and the H-bond distances or strengths of the salicylideneanilines. The crystallographic $\mathrm{N} \cdots$ O distance of $2.615 \AA$ measured for salicylideneaniline [40], which corresponds to $D_{0(\mathrm{ON})}=2.3 \mathrm{kcal} / \mathrm{mol}\left(D_{\mathrm{e}(\mathrm{ON})}=3.0 \mathrm{kcal} /\right.$ $\mathrm{mol})$, represents the neutral keto form of MSA. However, the zwitterionic enol form should be associated with a stronger

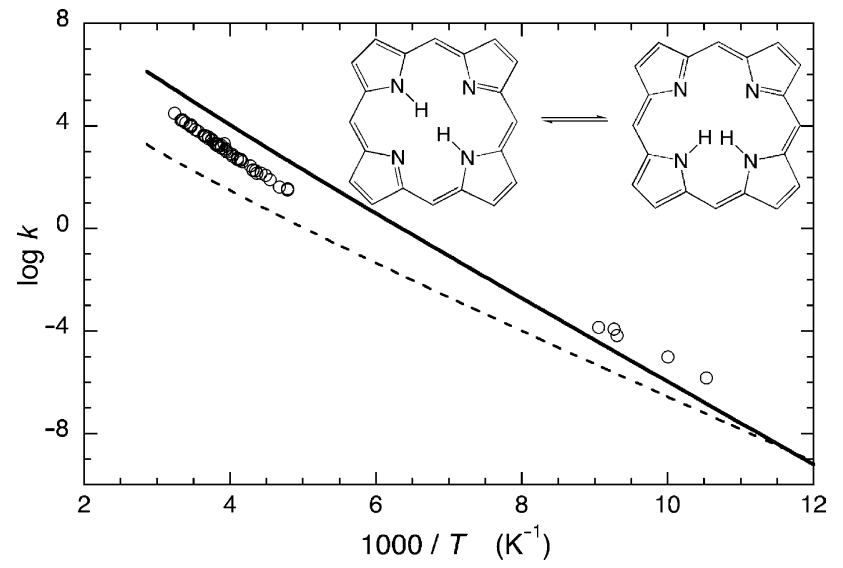

Fig. 2. Arrhenius plot for the thermal tautomerism of $\mathrm{H}_{2} \mathrm{P}$. The full and dotted lines represents LS-ISM/scTST and LS-ISM/Eckart calculations, respectively.
H-bond energy. In the absence of experimental information on the zwitterionic enol, and based on the similarity of lengths and strengths between the H-bonds in the neutral keto form and in water, we assume that strength of the $\mathrm{H}$-bond in the zwitterionic form is $D_{0\left(\mathrm{O}^{+} \mathrm{N}^{-}\right)}=6.4 \mathrm{kcal} / \mathrm{mol}\left(D_{\mathrm{e}\left(\mathrm{O}^{+} \mathrm{N}^{-}\right)}=\right.$ $8.8 \mathrm{kcal} / \mathrm{mol}$ ), which corresponds to $l_{\mathrm{O}^{+} \mathrm{N}^{-}}=2.44 \mathrm{~A}$. With these H-bonds, the data in Table $1, \Delta E^{0}=-0.5 \mathrm{kcal} / \mathrm{mol}$ and $E^{*}=2.57 \mathrm{eV}$ [12], we calculate $m=2.206$ and $k_{\mathrm{ISM}}=2.9 \times$ $10^{11} \mathrm{~s}^{-1}$ for MSA at $77 \mathrm{~K}$, which should be compared with the experimental value $k_{\exp }=2.8 \times 10^{11} \mathrm{~s}^{-1}$ [12]. The calculation involves a semiclassical tunnelling correction of $1.8 \times 10^{6}$. The agreement with the experimental value is better than expected for such a simple model, but it is nevertheless revealing of its capabilities to estimate the rates of ultra-fast ESIPT. The LSISM/Eckart rates at $77 \mathrm{~K}$ are one order of magnitude smaller than those of the LS-ISM/scTST method.

It is interesting to remark that, according to LS-ISM/scTST calculations, the thermodynamic difference between MSA and ClSA, does not suffice to justify their reactivity difference. Experimentally the rates increase by a factor of 4.5 , but the rates calculated with $\Delta E^{0}=-0.5$ or $-1.0 \mathrm{kcal} / \mathrm{mol}$ differ by a factor of 2.2 only. Other factors, namely differences in H-bond strengths, may contribute to the reactivity difference.

The proton, or $\mathrm{H}$-atom, transfer rates in the triplet state of HBO present several interesting features, that must be accommodated by reactivity models. First, at $77 \mathrm{~K}$ they are 5 orders of magnitude slower than the ESIPT in MSA, which has a similar exothermicity and involves the same reactive bonds. We interpret this reactivity difference in terms of the biradicaloid versus zwitterionic structures involved, and illustrated in Scheme 1. Second, the rates decrease from 200 to $60 \mathrm{~K}$, but then remain temperature independent down to $2 \mathrm{~K}$. We apply both semiclassical tunnelling corrections and Eckart barrier permeabilities to explore the full extent of this temperature (in)dependence. Finally, the kinetic isotope effect increases from 50 at $200 \mathrm{~K}$ to 1700 at $80 \mathrm{~K}$, and then remain independent of the temperature down to $2 \mathrm{~K}$.

Following the reasoning discussed above, these triplet-state reactions should be regarded as $\mathrm{H}$-atom transfers between biradicaloid species. The $\mathrm{H}$-bond lengths, energies and vibrational frequencies of both keto and enol forms should be similar to those of the neutral keto form of MSA in the groundstate, that the LS potential represents by $l_{\mathrm{NO}}=2.62 \AA$, $D_{0(\mathrm{NO})}=2.3 \mathrm{kcal} / \mathrm{mol}$ and $\varpi_{\mathrm{ON}}=230 \mathrm{~cm}^{-1}$. Additionally, the electrophilicity index of the biradical should be identical to that of the radical, and using the data on Table 1, we obtain $m=$ 1.714. The other parameters employed in the calculations are identical to those employed for the salicylideneanilines, except that experimentally $\Delta E^{0}$ decreases from 0 to $-0.4 \mathrm{kcal} / \mathrm{mol}$ as the temperature also decreases [16]. Fig. 3 presents the results of LS-ISM/scTST and LS-ISM/Eckart calculations using $\Delta E^{0}=-0.4 \mathrm{kcal} / \mathrm{mol}$. The LS-ISM/scTST calculations with $\Delta E^{0}=0$ give rates that are less than a factor of two smaller in the high-temperature range, where they best correspond to the experimental data, and do not change the overall representation of the data in Fig. 3. The LS-ISM/scTST method fails to reproduce the experimental rates below $100 \mathrm{~K}$ with any 


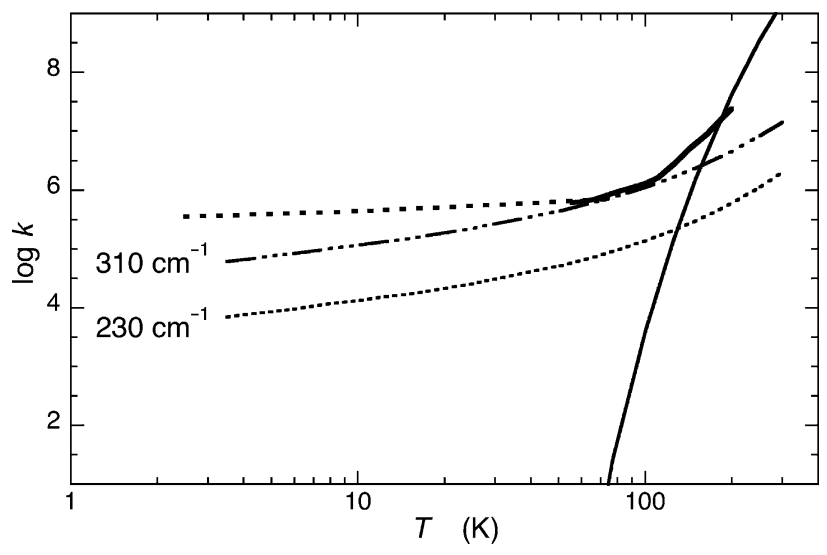

Fig. 3. Temperature-dependence of the keto-enol tautomerism in the tripletstate of HBO. Thick lines represent experimental data and thin lines represent calculations. The full and dotted lines represents LS-ISM/scTST and LSISM/Eckart calculations, respectively, with $\varpi=230 \mathrm{~cm}^{-1}$, and the dash-dotted line represents LS-ISM/Eckart calculations with $\varpi=310 \mathrm{~cm}^{-1}$.

meaningful set of parameters. On the other hand, below this temperature, LS-ISM/Eckart rates reproduce reasonably well the trends of the experimental data.

Preiffer and co-workers analysed the vibrational spectra of HBO and suggested that the vibrational mode observed at $310 \mathrm{~cm}^{-1}$ contributes substantially to the reaction path in the excited state along which the proton transfer proceeds [41]. This vibrational mode corresponds to the in-plane relative motion of the ring groups, during which the nitrogen and oxygen atoms approach each other [41], and is analogous to our intramolecular H-bond. It is reassuring that vibrational frequency is in good agreement with the value of $\varpi_{\mathrm{ON}}$ estimated by the LS potential, but it is even better to fit the LS potential to the observed vibrational mode and obtain the H-bond length and energy from that fit. This procedure yields, for $\varpi_{\mathrm{ON}}=310 \mathrm{~cm}^{-1}, l_{\mathrm{NO}}=$ $2.58 \AA$ and $D_{0(\mathrm{NO})}=2.9 \mathrm{kcal} / \mathrm{mol}$. The $\mathrm{H}$-atom transfer rates calculated with this LS potential are also represented in Fig. 3 and are in remarkable agreement with the experimental data. For example, even at $3.5 \mathrm{~K}$ we calculate $k_{\mathrm{tun}}=6.2 \times 10^{4} \mathrm{~s}^{-1}$, and the biexponential decays experimentally observed correspond to rates of $(1.7 \pm 0.2) \times 10^{5} \mathrm{~s}^{-1}$ and $(7 \pm 1) \times 10^{5} \mathrm{~s}^{-1}$ [16].

Following the LS-ISM/scTST method, the KIE increase only from 10 at $200 \mathrm{~K}$ to 16 at $150 \mathrm{~K}$, which should be compared with the experimental isotope effects of 50 and 100 at these temperatures [14]. The semiclassical approximation both underestimates the rates and the KIE at low temperatures. On the other hand, the Eckart barrier permeabilities give relatively constant and large KIE at low temperatures. For example, with $\varpi_{\mathrm{ON}}=310 \mathrm{~cm}^{-1}$, LS-ISM/Eckart calculations give a KIE of 47,000 at $100 \mathrm{~K}$, where it should be $1,700 \pm 300$ [16]. In view of the simplicity of the reaction coordinate and of the tunnelling corrections, it is remarkable how the calculations reproduce the 5 order of magnitude reactivity difference between ESIPT in the singlet state of MSA and the H-atom transfer in the triplet state of $\mathrm{HBO}$, as well as the temperature dependence of the latter reaction down to $3.5 \mathrm{~K}$. The success of these methods is that of the LS-ISM reaction path. The paths of these two reactions are compared in Fig. 4. The lower barrier of

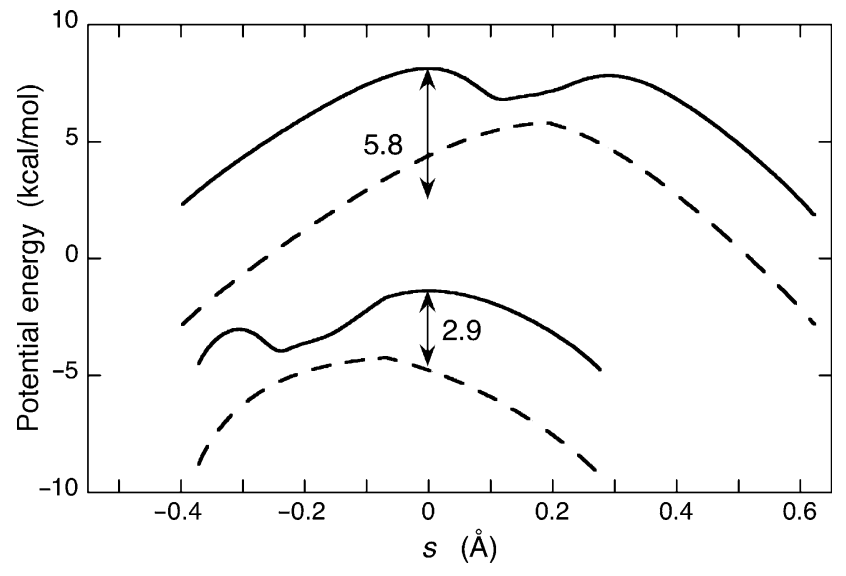

Fig. 4. Vibrationally-adiabatic (full lines) and classical (dashed lines) LS-ISM reaction paths for proton and $\mathrm{H}$-atom transfers using the data on MSA and HBO. The lower curves correspond to MSA and their displacement with the respect to the upper curves of $\mathrm{HBO}$ is due to the strength of the $\mathrm{H}$ bond in the reactants.

ESIPT in MSA is due to its larger electrophilicity index and to the stronger $\mathrm{H}$ bond in the reactants. Additionally, the $\mathrm{H}$-atom transfer in $\mathrm{HBO}$ has a steeper barrier because of the repulsion between the end atoms of the H-bond.

\section{Conclusions}

The LS-ISM method was employed to calculate unidimensional reaction paths for the thermal tautomerism of $\mathrm{H}_{2} \mathrm{P}$, the ESIPT in MSA, and the keto-enol tautomerism in the triplet state of HBO. LS-ISM is a semi-empirical method that does not require the fitting of any parameters to the kinetic data of these systems. The rate constants were calculated using the transition-state theory and the semiclassical approximation to tunnelling corrections or, alternatively, fitting an Eckart barrier to the LS-ISM reaction path and calculating its permeability. As the temperature is reduced below $100 \mathrm{~K}$, the classical TST rates became very low and the semiclassical tunnelling corrections become very high. Both these factors are very sensitive to the details of the LS-ISM reaction path, and their product is unreliable at such low temperatures. On the other hand, the Eckart barrier permeability has an analytical solution and its stability was observed even at $2 \mathrm{~K}$. Using the LSISM/scTST method to calculate the rates down to $100 \mathrm{~K}$ and the LS-ISM/Eckart method for lower temperatures, it was possible to obtain a good agreement between calculated and experimental rates. This agreement was made possible by the distinction between a proton transfer, in the singlet state of MSA, and a H-atom transfer, in the triplet state of HBO.

\section{Acknowledgements}

We thank Fundação para a Ciência e Tecnologia (Portugal) and FEDER for financial support; project no. POCTI/QUI/47267/2002. MB thanks the financial support by FCT through grant $\mathrm{BD} / 1332 / 2000$. 


\section{References}

[1] R.P. Bell, The Tunnel Effect in Chemistry, Chapman \& Hall, London, 1980.

[2] B.C. Garrett, D.G. Truhlar, J. Phys. Chem. 83 (1979) 2921.

[3] L.G. Arnaut, A.A.C.C. Pais, S.J. Formosinho, M. Barroso, J. Am. Chem. Soc. 125 (2003) 5236.

[4] M. Barroso, L.G. Arnaut, S.J. Formosinho, Chem. Phys. Chem. 6 (2005) 363.

[5] E.R. Lippincott, R. Schroeder, J. Chem. Phys. 23 (1955) 1099.

[6] T.J. Butenhoff, C.B. Moore, J. Am. Chem. Soc. 110 (1988) 8336.

[7] J. Braun, M. Schlabach, B. Wehrle, M. Köcher, E. Vogel, H.-H. Limbach, J. Am. Chem. Soc. 116 (1994) 6593.

[8] Z. Smedarchina, M.Z. Zgierski, W. Siebrand, P.M. Kozlowski, J. Chem. Phys. 109 (1998) 1014.

[9] D.L. Maity, R.L. Bell, T.N. Truong, J. Am. Chem. Soc. 122 (2000) 897.

[10] D.K. Maiy, T.N. Truing, J. Porphyrins Phthalocyanines 5 (2001) 289.

[11] E. Hadjoudis, I.M. Mavridis, Chem. Soc. Rev. 33 (2004) 579.

[12] T. Sekikawa, T. Kobayashi, T. Inabe, J. Phys. Chem. A 101 (1997) 644.

[13] K.H. Grellmann, A. Mordzinski, A. Heinrich, Chem. Phys. 136 (1989) 201.

[14] W. Al-Soufi, K.H. Grellmann, B. Nickel, J. Phys. Chem. 95 (1991) 10503.

[15] H. Eisenberger, B. Nickel, A.A. Ruth, W. Al-Soufi, K.H. Grellmann, M. Novo, J. Phys. Chem. 95 (1991) 10509.

[16] B. Nickel, K.H. Grellmann, J.S. Stephan, P.J. Walla, Ber. Bunsen-Ges. Phys. Chem. 102 (1998) 436.

[17] M.F. Rodrigues Prieto, B. Nickel, K.H. Grellmann, A. Mordzinski, Chem. Phys. Lett. 146 (1988) 387.

[18] H. Tukada, P.R. Bangal, N. Tamai, Y. Yokoyama, J. Mol. Struct. (Theochem) 724 (2005) 215.

[19] A. Douhal, F. Lahmani, A.H. Zewail, Chem. Phys. 207 (1996) 477.
[20] D.G. Truhlar, A.D. Isaacson, R.T. Skodje, B.C. Garrett, J. Phys. Chem. 86 (1982) 2252

[21] M. Barroso, L.G. Arnaut, S.J. Formosinho, Submitted for publication.

[22] H.S. Johnston, C. Parr, J. Am. Chem. Soc. 85 (1963) 2544.

[23] L. Pauling, J. Am. Chem. Soc. 69 (1947) 542.

[24] R.G. Parr, L.v. Szentpály, S. Liu, J. Am. Chem. Soc. 121 (1999) 1922.

[25] M. Barroso, L. Arnaut, S.J. Formosinho, J. Photochem. Photobiol. A Chem. 154 (2002) 13.

[26] E.B. Wilson Jr., J. Chem. Phys. 7 (1939) 1047.

[27] R. Triolo, A.H. Narten, J. Chem. Phys. 63 (1975) 3624

[28] D.M. Carey, G.M. Korenowski, J. Chem. Phys. 108 (1998) 2669.

[29] G.E. Walrafen, M.R. Fisher, M.S. Hokmabadi, W.-H. Yang, J. Chem. Phys. 85 (1986) 6970.

[30] R. Schroeder, E.R. Lippincott, J. Phys. Chem. 61 (1957) 921.

[31] B.C. Garrett, D.G. Truhlar, R.S. Grev, G.S. Schatz, R.B. Walker, J. Phys. Chem. 85 (1981) 3806.

[32] C. Eckart, Phys. Rev. 35 (1930) 1303.

[33] S.J. Formosinho, J. Chem. Soc. Faraday Trans. 270 (1974) 605.

[34] S.J. Formosinho, L.G. Arnaut, Adv. Photochem. 16 (1991) 67.

[35] Z. Wang, P.N. Day, R. Pachter, J. Chem. Phys. 108 (1998) 2504.

[36] David R. Lide (Ed.). Handbook of Chemistry and Physics. CRC Press, 2001.

[37] B. Cronin, M.G.D. Nix, R.H. Qadiri, M.N.R. Ashfold, PCCP 6 (2004) 5031.

[38] A.J. Gianola, T. Ichino, R.L. Hoenigman, S. Kato, V.M. Bierbaum, W.C. Lineberger, J. Phys. Chem. A 108 (2004) 10326.

[39] S.J. Formosinho, L.G. Arnaut, J. Photochem. Photobiol. A Chem. 75 (1993) 21.

[40] F. Arod, M. Gardon, P. Pattison, G. Chapuis, Acta Crystallogr. C 61 (2005) 317.

[41] M. Pfeiffer, K. Lenz, T. Elsaesser, T. Steinke, J. Raman Spectrosc. 29 (1997) 61-72. 